\title{
Focal transcranial magnetic stimulation and response bias in a forced-choice task
}

\author{
Joaquim P Brasil-Neto, Alvaro Pascual-Leone, Josep Valls-Solé, Leonardo G Cohen, \\ Mark Hallett
}

\begin{abstract}
The effects of transcranial magnetic stimulation were studied on the performance of a warned, forced-choice response time task by normal adults. The task consisted of extension of the index finger in response to the click produced by the discharge of the magnetic coil (go-signal). The subjects were asked to choose the right or left finger only after the go-signal was delivered. Single magnetic stimuli were delivered to the prefrontal or motor area, and in the control situation, away from the head. Magnetic stimulation affected hand preference only when it was delivered to the motor area. With stimulation of this area, subjects more often chose the hand contralateral to the site stimulated with response times that were mainly less than $200 \mathrm{~ms}$. With longer response times (between 200 and $1100 \mathrm{~ms}$ ), magnetic stimulation had no effect on hand preference regardless of the site stimulated. Stimulation of prefrontal areas yielded results similar to the control situation. These results suggest that response bias in this paradigm is caused by an effect of magnetic stimulation on neural structures within, or closely related to, the motor areas of the brain. Although the response bias was clear and predictable, the subjects were unaware of its existence. It is possible to influence endogenous processes of movement preparation externally without disrupting the conscious perception of volition.
\end{abstract}

(F Neurol Neurosurg Psychiatry 1992;55:964-966)

Human Cortical Physiology Unit Human Motor Control Section, Medical Neurology Branch, National Institute of Neurological Disorders and Stroke, National Institutes of Health, Bethesda, Maryland, USA J P Brasil-Neto A Pascual-Leone J Valls-Solé L G. Cohen $M$ Hallett

Correspondence to: Dr Hallett, Building 10 Room 5N226, NINDS, NIH, Bethesda, Maryland 20892, USA

Received 8 August 1991 and in revised form 17 December 1991. Accepted 2 January 1992 and prefrontal areas on response bias in a forced-choice task, with emphasis on the relation between response time, hand preference, and specific sites of transcranial stimulation.

\section{Methods}

We studied 4 normal subjects ( 3 men and 1 woman), aged 26 to 45 years, who gave written informed consent for the study. The protocol was approved by the Institute's clinical investigational review committee. Handedness was assessed with the Edinburgh Handedness Inventory. ${ }^{11}$

The subjects sat on a chair with their forearms supported on a horizontal surface. They were instructed to extend either their right or left index finger at will after delivery of the stimulus, but to decide which finger to move only after hearing the click produced by the Cadwell MES-10 magnetic stimulator (gosignal). An auditory warning was delivered 3 to 5 seconds before the go-signal. The subjects were specifically instructed to avoid repetitive selection of the same hand and to avoid constant alternation of the hands. A pair of surface electrodes (DISA 13K60) was placed on the skin overlying the belly of the extensor indicis proprii muscles of the right and left hands. EMG activity was recorded by a DISA 1500 electromyograph, with a bandpass of $50 \mathrm{~Hz}$ to $2 \mathrm{kHz}$ and sensitivity ranging from 50 to $1000 \mu \mathrm{V} /$ division.

Threshold intensity, or the minimal stimulus intensity required to produce at least one motor evoked potential (MEP) of $25 \mu \mathrm{V}$ in amplitude in a series of 10 stimuli delivered to the motor area with an 8-shaped coil, was determined. The stimulus intensity was always $5 \%$ below the threshold intensity (for example, if the threshold intensity was $50 \%$ of the maximal output of the stimulator, the stimulus was delivered at $45 \%$ of the output).

Each subject had a training session in which the task was practised with a series of 40 stimuli delivered away from the head; no data were collected during this session. The subject then performed the task while two series of 60 to 80 stimuli were delivered to the $\mathrm{Fz}$ position, to a position $5 \mathrm{~cm}$ lateral to $\mathrm{Fz}$ on both sides of the head, and to the optimal positions for producing MEPs in the target muscles (that is, the "motor areas" bilaterally). Response times were measured on the EMG tracings and were arbitrarily classified as extra-short $(<200 \mathrm{~ms})$, short $(200-500 \mathrm{~ms})$, medium (500-800 ms), long (800-1100 ms), and extra-long (> $1100 \mathrm{~ms})$. 
For control trials, two series of 80 stimuli were delivered away from the head.

Response times were expressed as the mean (SD) or as the number of occurrences in each class of response time (extra-short, short, medium, long, extra-long). The distribution of response times as a function of the scalp area stimulated and across subjects was studied with one way analysis of variance (ANOVA). One way ANOVA was also used to analyse the effects of the scalp area stimulated and of handedness on the side ispilateral or contralateral to the cerebral hemisphere stimulated. Two way ANOVA was used to analyse the interdependence of the scalp area stimulated, the side of response, and the response time.

\section{Results}

The response times varied widely in all subjects; an example of the variability is shown in fig 1 . The distribution of the extra-short, short, medium, and long response times was similar in trials with and without magnetic stimulation, but the majority $(80 \%)$ of the extra-short responses occurred when the motor area was stimulated (fig 2). Hand preference was affected only by magnetic stimulation of the motor area. With stimulation of this area, subjects more often chose the hand contralateral to the site stimulated. There was an excess $(p=0.05)$
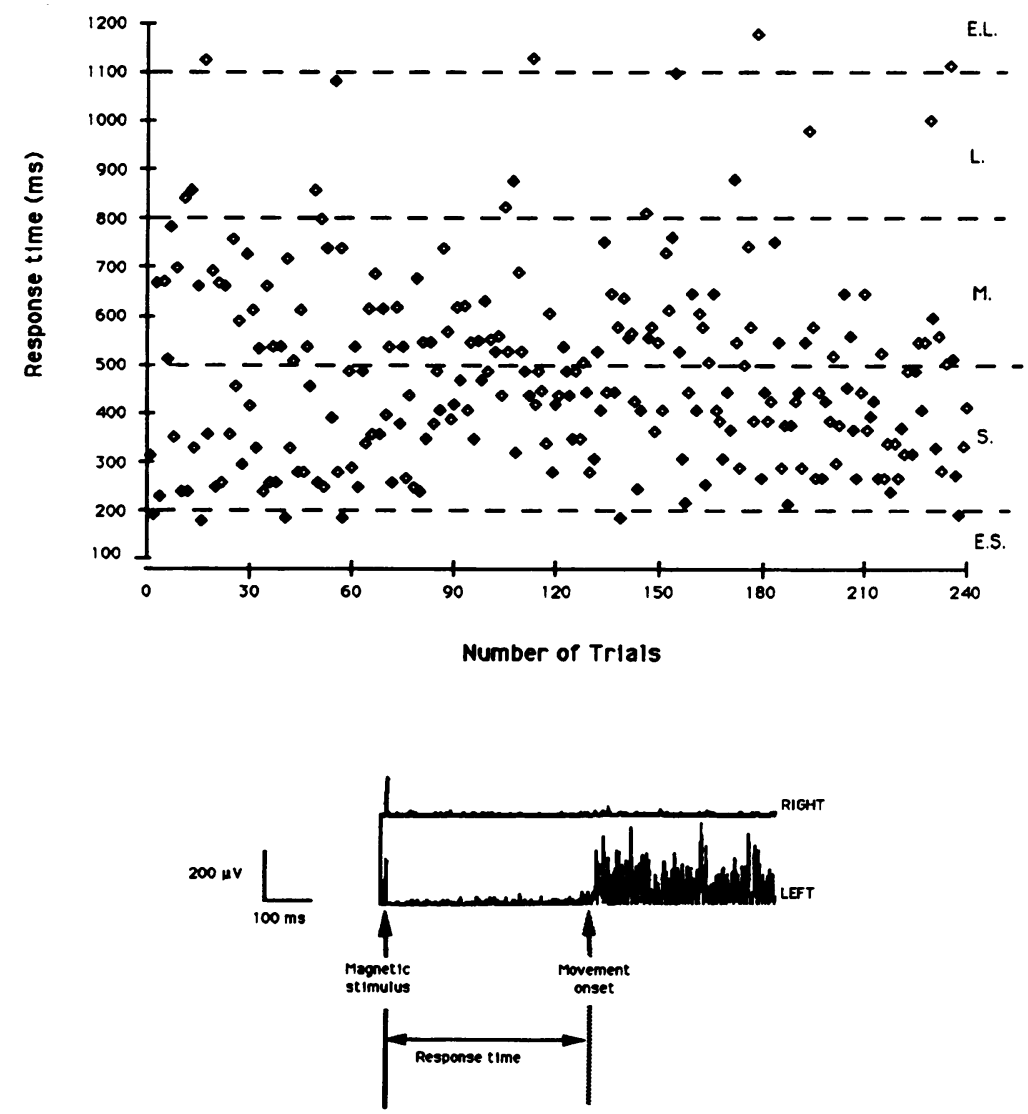

Figure 1 Upper panel: Response times in a single patient. Open squares represent trials with the coil away from the head. Solid squares represent trials with stimulation of different scalp areas. The different response times (EL, extra-long; $L$, long; $M$, medium; $S$, short; ES, extra-short) are separated by dashed lines. Lower panel: rectified EMG tracing from the same patient. of contralateral responses with stimulation of the motor areas. However, they were not aware of any effect of the stimulus on their response pattern. The prevalence of contralateral responses to stimulation of the motor area was accounted for mainly by extra-short response times ( $p<0.001$ ) (fig 3 ).

Three subjects had a tendency for faster response times with the dominant hand. However, the prevalence of contralateral responses of extra-short latency with stimulation of the motor cortex was independent of handedness or the cerebral hemisphere stimulated. The fourth subject was ambidextrous and showed no significant difference in response times between the two hands.

\section{Discussion}

In warned, simple reaction time paradigms, the subject is given all the necessary information to plan the response during the time between the warning and the go-signal (foreperiod). The experimental paradigm that we used is different, because after the go-signal, the subject was supposed to choose which side of the body to use in the response. The latency from the go-signal to the finger movement is a response time rather than a reaction time, since the subjects were not required to react as fast as possible. The relation between cortical stimulation and contralateral responses of extra-short latency may be explained by the response preparation model described elsewhere. ${ }^{912}$ In the trials in which the magnetic stimulus had an effect, it is likely that activation of the response channel during the foreperiod came very close to the threshold, with the result that the magnetic stimulus aided the production of the response. Previous observations that a subthreshold magnetic stimulus over the motor cortex significantly shortened the reaction time for tasks performed with the contralateral arm have been similarly interpreted. ${ }^{9}$ Magnetic stimulation over the motor area could theoretically precipitate response channel discharges through two different mechanisms: disinhibition or direct activation of response channels. In other words, it could either reduce the threshold of response channel activation or increase the rate of activity build up in the response channel, causing a fixed threshold to be reached earlier.

Ammon and Gandevia ${ }^{10}$ stated that magnetic stimulation may influence the selection of motor programs at levels upstream of the motor cortex. However, they used a large coil that delivered a nonfocal stimulus (for a description of electric fields induced by different coil designs, see Cohen et al ${ }^{13}$ ), making it likely that the motor areas were also stimulated when the coil was positioned over the prefrontal areas of their subjects. They postulated that "the exact cellular elements responsible for the change in hand preference are presumably anterior to, or can be influenced anterior to, the motor cortex." From our results, it appears that the site of action is restricted to the motor areas of the cerebral hemispheres. The magnetic stimulus largely 


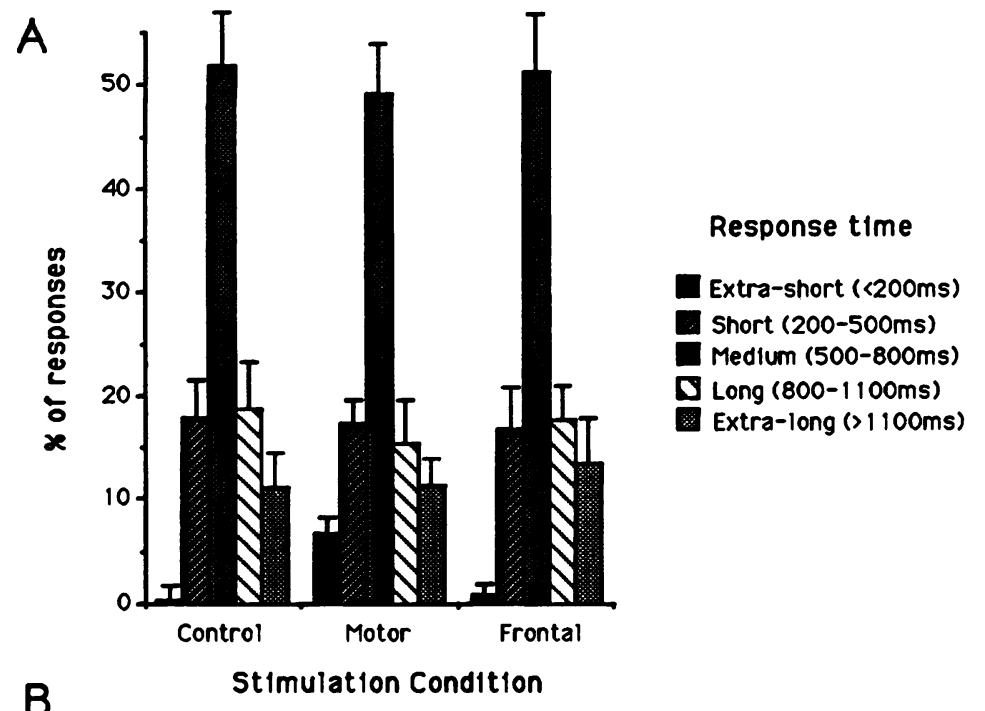

в

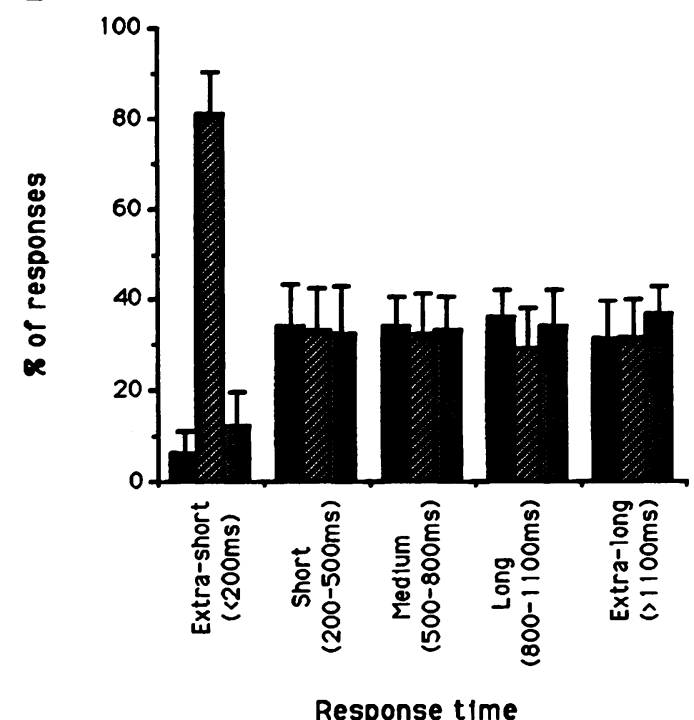

Figure 2 A) The distribution of response times is similar for the different scalp areas stimulated; however, there are more extra-short response times with stimulation of the motor areas. B) Most of the extra-short response times were obtained with stimulation of the motor areas.

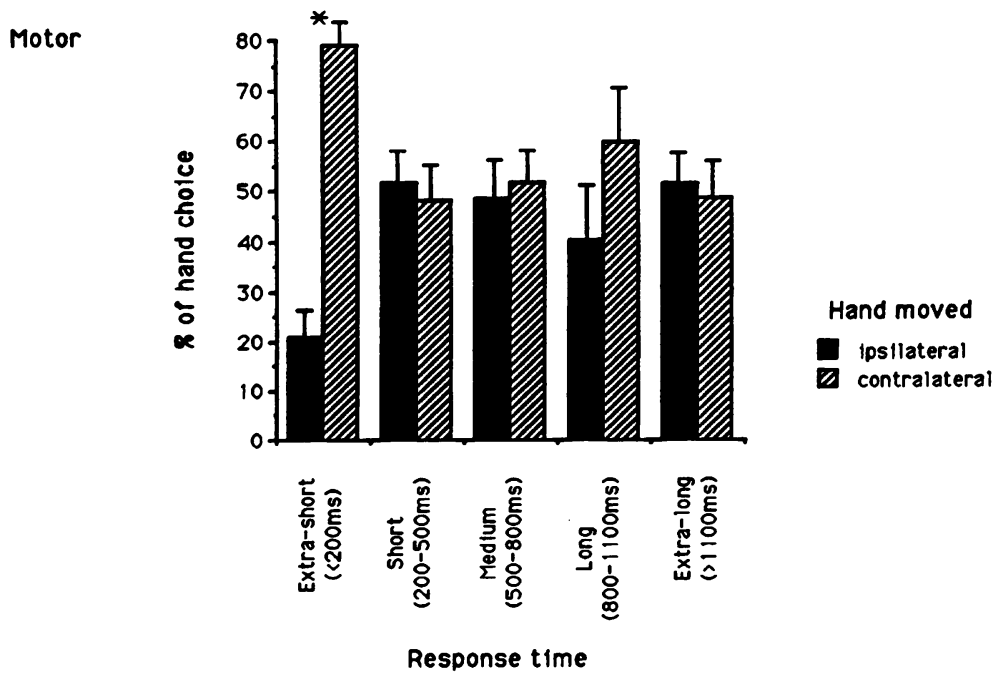

Figure 3 Frequency of ipsilateral and contralateral response times to magnetic ${ }_{* p}<0.001$. influenced hand choice when it also decreased the response time. Using more focal stimulation, we showed that the effect of a single magnetic stimulus on selection of specific motor programmes is mediated by neural structures within, or closely related to, the motor areas of the brain.

The fact that the subjects were unaware of any influence of the magnetic stimulus on their choice of response shows that it is possible to influence movement preparation processes externally without disrupting the conscious perception of volition. Libet et al ${ }^{14}$ studied the temporal relation between the recordable cerebral activity preceding a voluntary, fully endogenous motor act (the readiness potential) and the reportable time of appearance of the subjective experience of "wanting" or intending to act. They found that the onset of cerebral activity preceded by at least several hundred milliseconds the reported time of conscious intention to act. Our results agree with those findings and suggest that conscious perception of willing a particular action is preceded, and possibly generated, by cerebral processes that can be influenced by magnetic stimulation. Since conscious perception and the resulting movement can be consistently and predictably influenced by magnetic stimulation of the motor areas, these early cerebral processes probably account for the generation of both the conscious perception of wanting to move and the corresponding movement.

1 Amassian VE, Cracco RQ, Maccabee PJ, Somasundaram $M$. Focal magnetic coil stimulation near human central sulcus exceptionally elicits localized contralateral parsulcus exceptionally elicits localized contral

2 Cohen LG, Topka HR, Cole RA, Hallett M. Leg paresthesias induced by magnetic brain stimulation in patients with thoracic spinal cord injury. Neurology 1991: 41:1283-8.

3 Cohen LG, Bandinelli S, Sato S, Kufta C, Hallett M. Attenuation in detection of somatosensory stimuli by transcranial magnetic stimulation. Electroencephalogr Clin Neurophysiol 1991:81:366-76.

4 Amassian VE, Cracco RQ, Maccabee PJ. A sense of movement elicited in paralyzed distal arm by focal magnetic coil stimulation of human motor cortex. Brain Res 1989:479:335-60.

5 Barlow HB, Kohn HL, Walsh EG. Visual sensations aroused by magnetic fields. Am F Physiol 1947;148:372-5.

6 Dunlap K. Visual sensations from the alternating magnetic field. Science 1911;33:68-71.

7 Magnusson LE, Stevens HC. Visual sensations created by a magnetic field. Philosophical Magazine 1914;28:188-207.

8 Day BL, Rothwell JC, Thompson PD, et al. Delay in the execution of voluntary movement by electrical or magexecution of voluntary movement by electrical or mag
netic brain stimulation in intact man. Brain 1989; netic brain

9 Pascual-Leone A, Valls-Solé J, Wassermann EM, BrasilNeto J, Cohen LG, Hallett M. Effects of focal transcranial magnetic stimulation on simple reaction time to acoustic, visual, and somatosensory stimuli. Brain 1992 (in press).

10 Ammon K, Gandevia SC. Transcranial magnetic stimulation can influence the selection of motor programmes. $\mathcal{F}$ Neurol Neurosurg Psychiatry 1990;53:705-7.

11 Oldfield RC. The assessment and analysis of handedness: the Edinburgh inventory. Neuropsychologia 1971;9: 97-113.

12 Gratton G, Coles MGH, Sirevaag EJ, Eriksen CW, Donchin E. Pre- and poststimulus activation of response channels: a psychophysiological analysis. $\mathcal{f}$ Exp Psychol [Hum Percept] 1988;14:331-44.

13 Cohen LG, Roth, BJ, Nilsson J, et al. Effects of coil design on delivery of focal magnetic stimulation: technical on delivery of focal magnetic stimulation: technical considerations.

14 Libet B, Gleason CA, Wright EW, Pearl DK. Time of conscious intention to act in relation to onset of cerebral of a freely voluntary act. Brain 1983:106:623-42. 